\title{
A CASE OF SPONTANEOUS DISSECTING ANEURYSM OF THE INTERNAL CAROTID ARTERY
}

\author{
BY \\ R. McD. ANDERSON* and M. M. SCHECHTER
}

From the National Hospital, Queen Square, London

Dissecting aneurysm of the internal carotid artery, without preceding trauma, is a rare event. We have been unable to find a previous case report.

One case of dissecting aneurysm of the internal carotid artery following head injury has been reported (Dratz and Woodhall, 1947), and two cases after carotid puncture for arteriography (Sirois, Lapointe, and Côté, 1954; de Grood, 1954) where the common carotid dissection extended into the internal carotid arteries. As an extension of a dissecting aneurysm of the aorta Shennan (1934) reported five cases.

The following case, apart from its rarity, is of both radiological and pathological interest.

\section{Case Report}

A.R. (N.H. 75263), a man, 41 years of age, was admitted to the National Hospital, Queen Square, on October 16, 1957, under the care of Dr. John Marshall.

The history obtained on admission was from an accompanying letter which stated that the patient had developed a right hemiparesis on the day before admission. The development of paresis was over a period of several hours. There was a past history of cystitis.

At the time of admission he was conscious but unable to speak or comprehend spoken commands. His eyes were deviated to the left and he appeared to have a right homonymous hemianopia. There was a right hemiparesis with weakness of the right side of the face. The lower limb was weaker than the upper limb. Tone was increased in both upper limbs and in the right lower limb. The tendon reflexes were more active on the right. A grasp reflex was present on the left side and the plantar responses were bilaterally extensor. There was a response to pin prick on both sides. The blood pressure was $149 / 95 \mathrm{~mm}$. Hg. No other abnormality was found on general examination.

Lumbar puncture soon after admission produced clear cerebrospinal fluid at a pressure of $160 \mathrm{~mm}$. of C.S.F., and it contained three lymphocytes per c.mm., $25 \mathrm{mg}$. of protein per $100 \mathrm{ml}$., and the C.S.F. Wassermann reaction was negative. The erythrocyte sedimentation rate was $51 \mathrm{~mm}$. in one hour and $56 \mathrm{~mm}$. in two hours.

The day after admission carotid arteriography was

* Nuffield Dominion Scholar from the University of Melbourne. carried out. The left common carotid artery was punctured below the level of the fourth cervical vertebra, and two injections, $8 \mathrm{ml}$. each, of $45 \%$ "hypaque" were made. The radiographs showed contrast medium in a normal-looking common carotid artery for the upper $4 \mathrm{~cm}$. of its course, and a normal-looking external carotid artery. In the internal carotid artery, however, about $1.5 \mathrm{~cm}$. from its origin there was a localized filling defect extending for approximately $5 \mathrm{~mm}$. Three centimetres above its origin the lumen tapered to a very narrow channel which reached its maximum degree of constriction at a point $4.5 \mathrm{~cm}$. from the origin of the artery. Approximately $6 \mathrm{~cm}$. from the origin of the artery the shadow of the contrast medium widened again and was seen to outline the intracranial course of the artery and its branches (Figs. 1a, b, and c), but inadequately. In order to outline the entire length of the left common carotid artery, the artery was punctured again as low down in the neck as possible, and two injections, $8 \mathrm{ml}$. each, of $45 \%$ "hypaque" were given. This demonstrated the lower part of the common carotid artery, which appeared normal, and the deformity of the internal carotid artery was again demonstrated. It was considered that the left internal carotid artery was partially occluded by atheroma and thrombus.

Anticoagulant therapy was begun but the patient became progressively more drowsy and three days after admission he developed a left third cranial nerve palsy. The left lateral ventricle was tapped through a burr hole. This showed cerebrospinal fluid under normal pressure. The patient died later the same day, October 19, 1957, four days after the onset of his illness.

\section{Necropsy}

Necropsy was carried out 43 hours after death and the principal findings were as follows:

General Examination.-The body was that of a well nourished, middle-aged male with no skin lesions other than those due to the surgical procedures. There were dense adhesions at the apex of the right lung. Subapical caseous nodules were present in the upper lobes of both lungs. The heart weighed $420 \mathrm{~g}$; the myocardium, endocardium, and valves were normal. There was mild patchy atheroma of the coronary arteries and of the aorta. The liver 
weighed 1,950 g., its cut surface pale and yellowish. The spleen weighed $200 \mathrm{~g}$. and the Malpighian corpuscles were more prominent than usual. The left kidney weighed $300 \mathrm{~g}$. and was about twice the size of the right kidney. On section the left kidney showed a normal architecture. The parenchyma of the right kidney was almost wholly replaced by circumscribed areas of caseous material. The wall of the right ureter was thickened and its lumen contained caseous material. The bladder wall was thickened and its mucosa inflamed. The seminal vesicles were enlarged, but the testes appeared to be normal.

Histological Examination.-The caseous nodules in the lungs, kidney, ureter, and seminal vesicles were tuberculous in appearance. Numerous tubercle bacilli were demonstrated in the renal lesions. Microscopically the prostate also contained caseous nodules. Gross fatty change was present in the liver. No amyloid change was demonstrated in the liver, spleen, or kidneys.
Brain.- The left hemisphere was enlarged and pale. It was softened over its anterior two-thirds. Temporal herniation was present on the left side. Coronal sections through the cerebrum showed softening of the left frontal and parietal lobes, including the cortex, white matter, and the basal ganglia in the distribution of the left anterior and middle cerebral arteries. In places there were petechial haemorrhages in the softened cortex. There was also some softening of the medial part of the right frontal lobe in the distribution of the right anterior cerebral artery. The lateral and third ventricles were displaced to the right side. The cranial part of the brain-stem was displaced caudally and scattered haemorrhages were present in the cranial part of the brain-stem. The circle of Willis was complete. The right anterior cerebral artery was narrow from its origin to the anterior communicating artery, and beyond this point it contained ante-mortem blood clot filling the lumen and adherent to the wall. The right posterior cerebral artery arose principally from the right

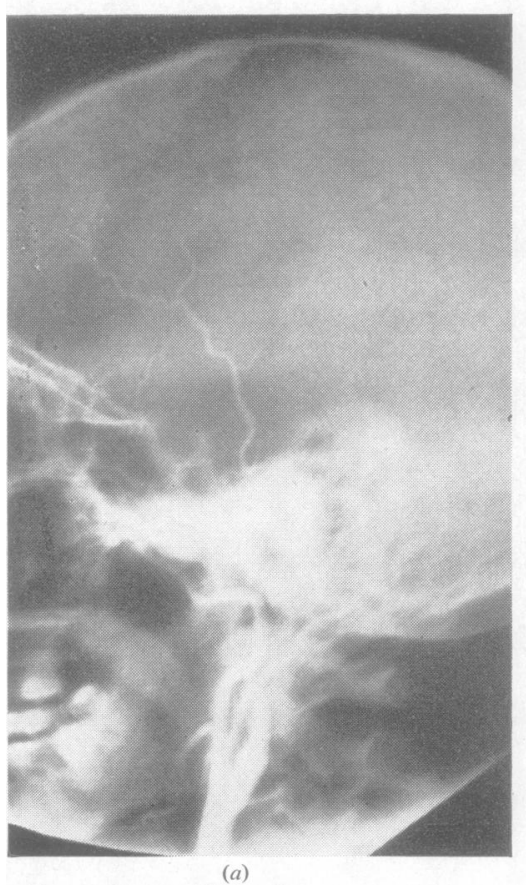

(a)

(a) The needle point, which is not shown, must be proximal to the end of the common carotid artery. The external carotid artery and its branches are outlined. The internal carotid artery tapers $3 \mathrm{~cm}$. above its origin.

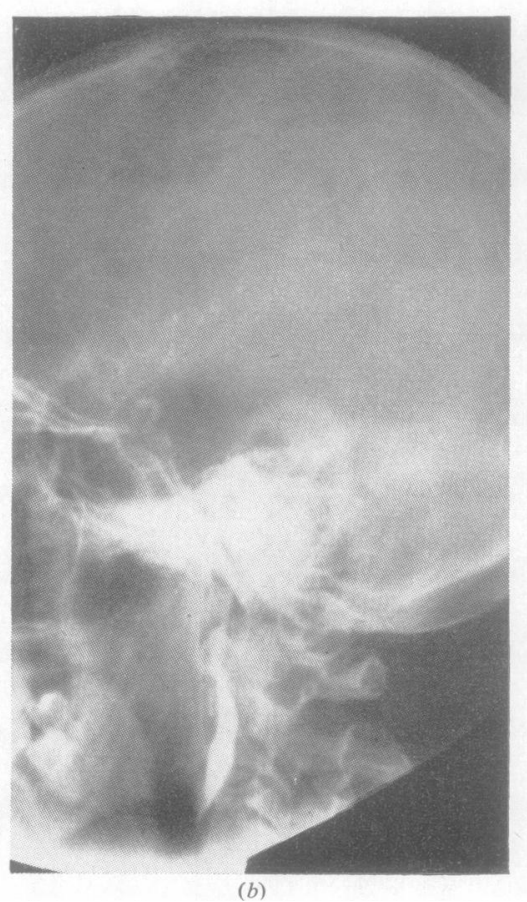

Fig. 1.-Left common carotid arteriogram.

(b) Film taken 1.5 seconds after film (a). Contrast medium still persists in the internal carotid artery but beyond the constriction the artery widens and contrast medium is present in the carotid siphon and its branches.

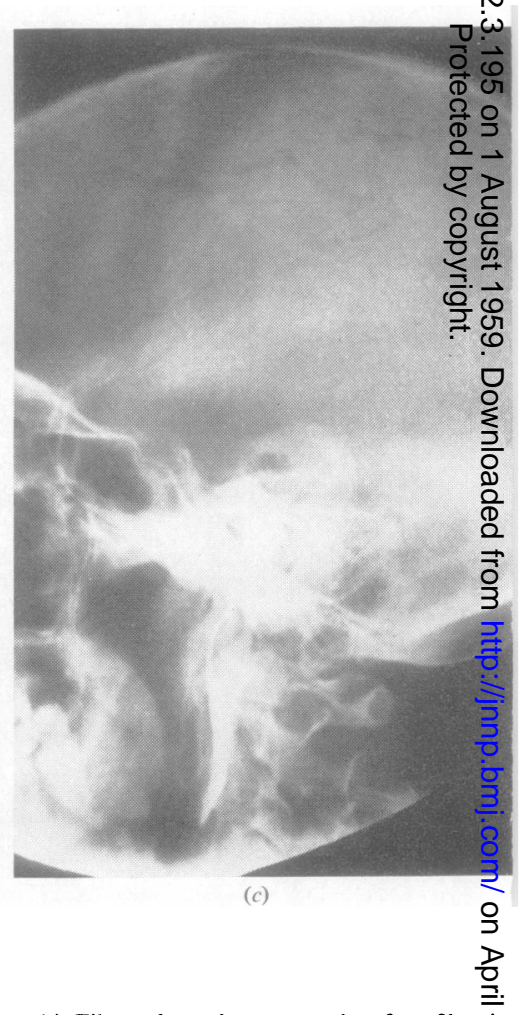

(c) Film taken three seconds after film niv Fig. (a). Contrast medium still persists in $\$$ H internal carotid artery. 


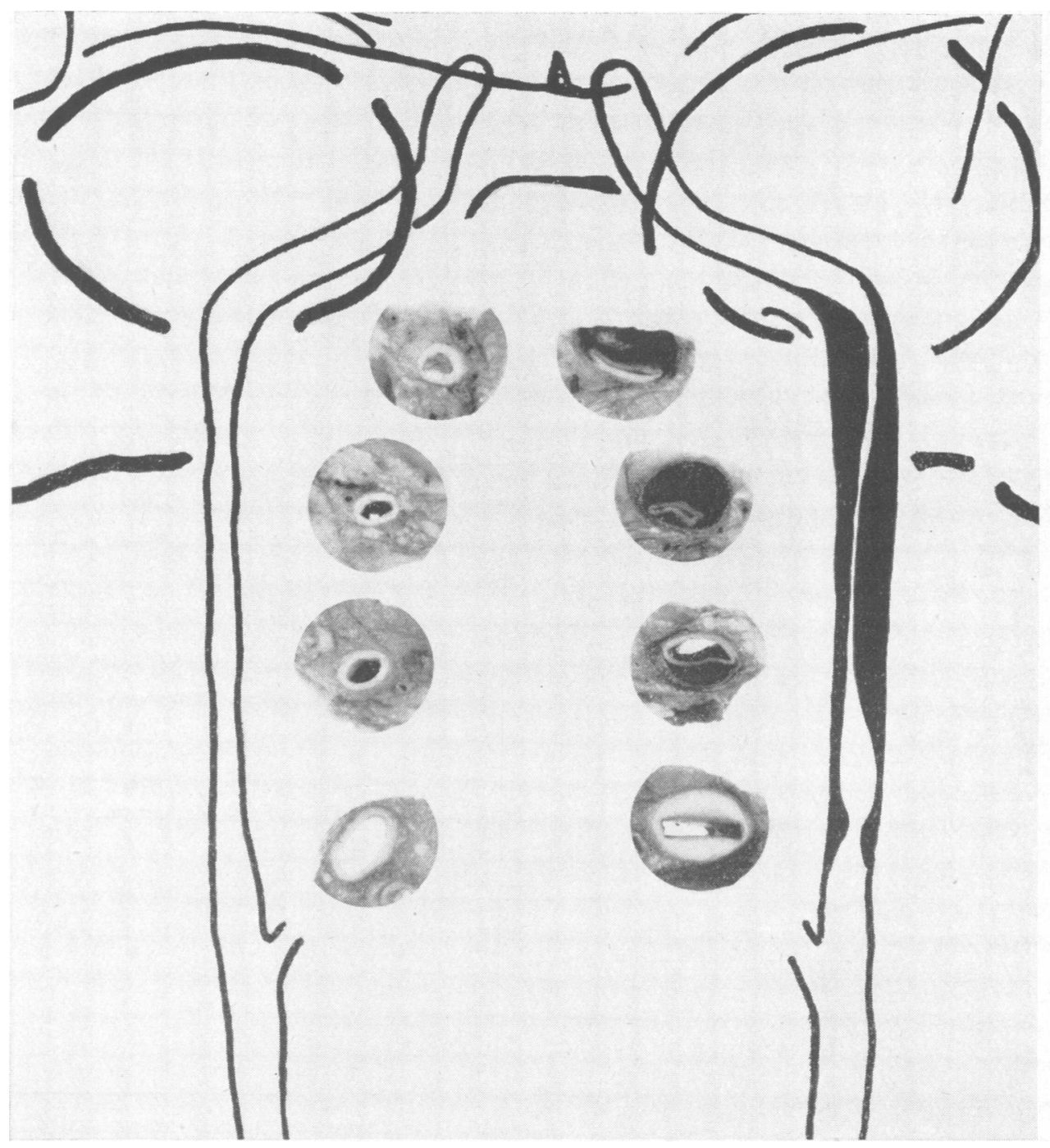

FIG. 2.-A combined diagram and photograph showing a reconstruction of the internal carotid arteries after cutting them into transverse sections. Some of these sections are shown opposite the appropriate portion of the artery (for description see text). Sections shown in Figs. 3, 4, 5, and 6 were taken from the sites indicated.

internal carotid artery, and only a narrow vessel connected it with the basilar artery. The walls of the major arteries appeared healthy.

Histological Examination of the Brain.-Softened areas from the right and left frontal lobes showed the changes usually seen in recent infarction. The neurones were shrunken. Polymorphonuclear cells and mononuclear cells were present in the parenchyma, particularly around the blood vessels and in the leptomeninges. Astrocytes appeared to be increased in number and there was endothelial hyperplasia of the blood vessels. Myelin in the softened areas stained poorly. The lesions in both frontal lobes appeared to be of approximately the same age.

Arteries of the Neck.-The carotid and vertebral arteries and arch of the aorta were removed en bloc with the cervical spine and base of the skull. After one week of fixation in formol saline the arch of the aorta, carotid arteries to the base of the skull, and vertebral arteries as far as their entry into the vertebral transverse processes were removed for 


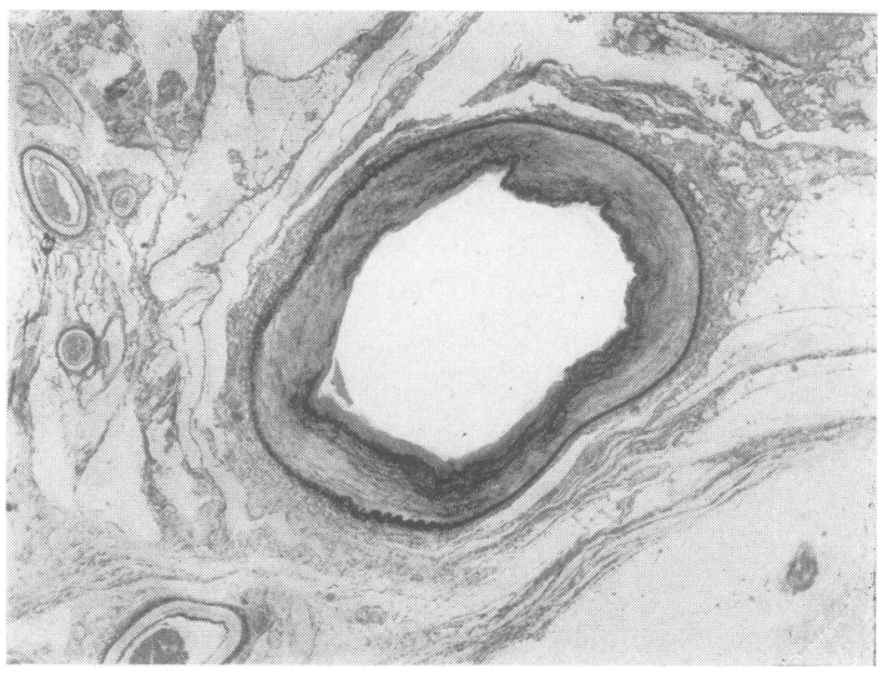

Fig. 3.-Transverse section through the right internal carotid artery showing the irregularity and deficiency of the elastica in the media. This section was taken a little above the level of the section from the left side (Fig. 4). The levels are indicated in Fig. 2. Weigert's elastic stain $(\times 12)$.

sectioning. The remaining bony parts were then decalcified in $5 \%$ nitric acid.

Horizontal sections were cut through the carotid and vertebral arteries.

The arch of the aorta, innominate artery, and the right common carotid artery were normal. There were two angiographic puncture wounds in the anterior wall of the left common carotid artery, and a moderate amount of blood staining of the surrounding muscle. This artery was otherwise normal.

The vertebral arteries were normal throughout their entire length. The right internal carotid artery was a little narrower than the left at its origin (right $7 \mathrm{~mm}$. diameter, left $8 \mathrm{~mm}$. diameter). Two centimetres above its origin it narrowed to $4 \mathrm{~mm}$. diameter and remained narrow as far as the base of the skull (Fig. 2) after which for the rest of its course it was normal.

One centimetre above its origin the lumen of the left internal carotid artery was diminished by opposing plaques of atheroma. This narrowing persisted for $5 \mathrm{~mm}$. and then the lumen returned to its previous diameter. At $3 \mathrm{~cm}$. above its origin the lumen of the artery was almost completely occluded by haemorrhage within its wall. For a short distance this dissecting haemorrhage was confined to the anterior wall of the artery, and above this it surrounded the whole lumen of the vessel. The intramural haemorrhage extended for between 3 and $4 \mathrm{~cm}$. to the point where the artery turned antero-medially beneath the floor of the middle fossa. Here there was an intimal tear. Beyond this point the artery appeared normal (Fig. 2).
Histological Examination of Arteries.-After embedding in paraffin and/or celloidin, transverse sections were cut through the arteries and stained with haematoxylin-eosin, haematoxylin-van Gieson, thionine blue, Weigert's elastic tissue stain, Verhoeff's elastic tissue stain, and Masson's trichrome.

The aortic wall showed a little cystic change in the media but was otherwise normal.

In the media of the left and right common carotid arteries there was a moderate increase of metachromatic material. The elastic tissue and muscle appeared normal. Atheroma was present.

The left external carotid artery also showed an increased amount of metachromatic material in the media.

The wall of the right internal carotid artery contained an increased amount of metachromatic material in its media and cystic change was well marked. The elastic tissue pattern was abnormal; there was less elastic tissue than usual, and the fibres appeared to be shorter and thicker than normal (Fig. 3). The muscle of the media appeared to be comparatively normal. Vascularization of the media was not evident.

The left internal carotid artery (Fig. 4) showed similar changes to those of the artery on the right, but in addition there was haemorrhage into the wall of the artery in the outer part of the media (Fig. 5). A longitudinal section through the artery at the lower end of this intramural haemorrhage showed a tear through the media and intima into the lumen of the vessel (Fig. 6). Thus there were intimal tears at both ends of the dissecting haemorrhage. The upper tear was mentioned previously in the 


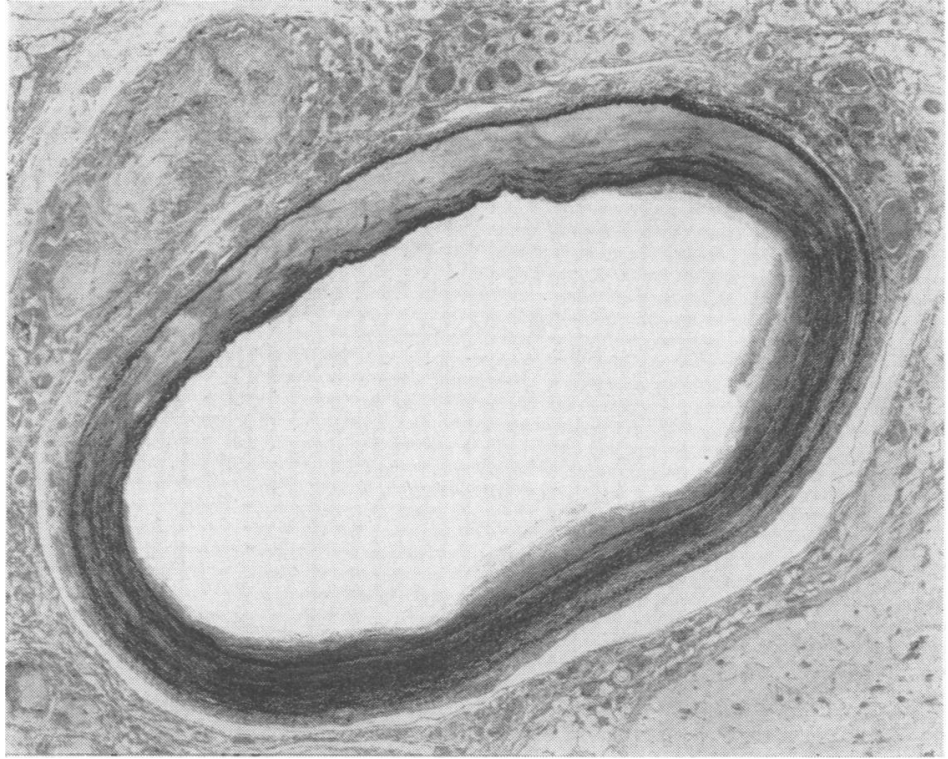

Fig. 4.-Transverse section through the left internal carotid artery just below the site of the dissecting haemorrhage in its wall. The posterior part of the wall in the upper part of the photomicrograph appears to be normal, but in the anterior wall the elastica of the media is deficient. Weigert's elastic stain $(\times 12)$.

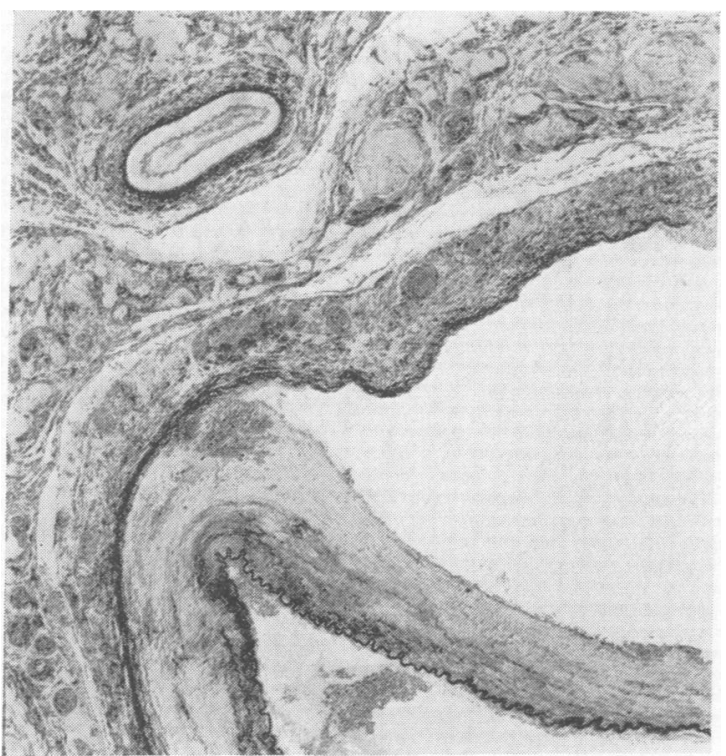

Fig. 5.-Transverse section of the left internal carotid artery a little above the lower end of the dissecting haemorrhage. Blood clot has fallen out of the media in preparing the section; however. the position of the blood clot is shown to be in the outer part of the media. The deficiency in the elastica of the media is well shown. Weigert's elastic stain $(\times 30)$.

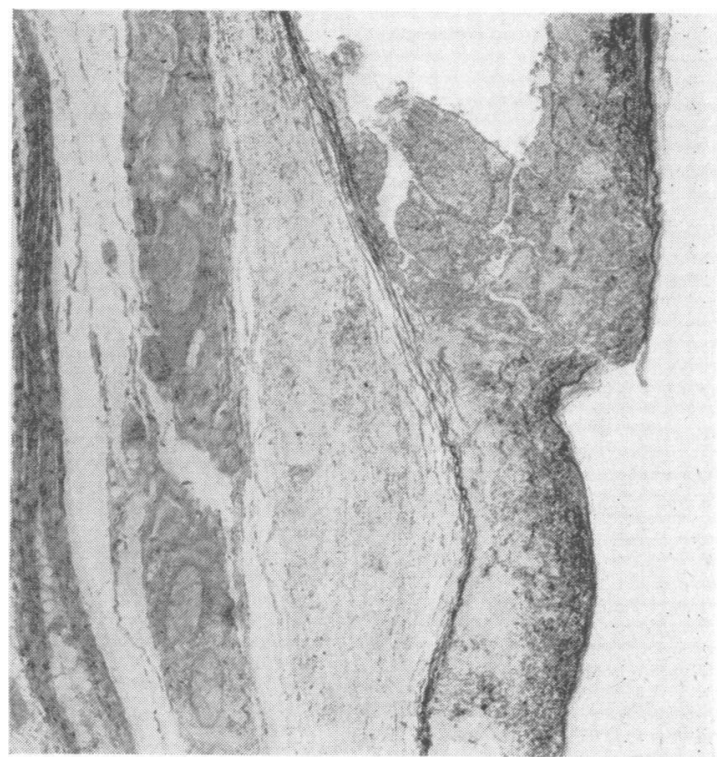

Fig. 6.-Longitudinal section through the lower end of the dissecting aneurysm to show the intimal tear. Weigert's elastic stain $(\times 27)$. 


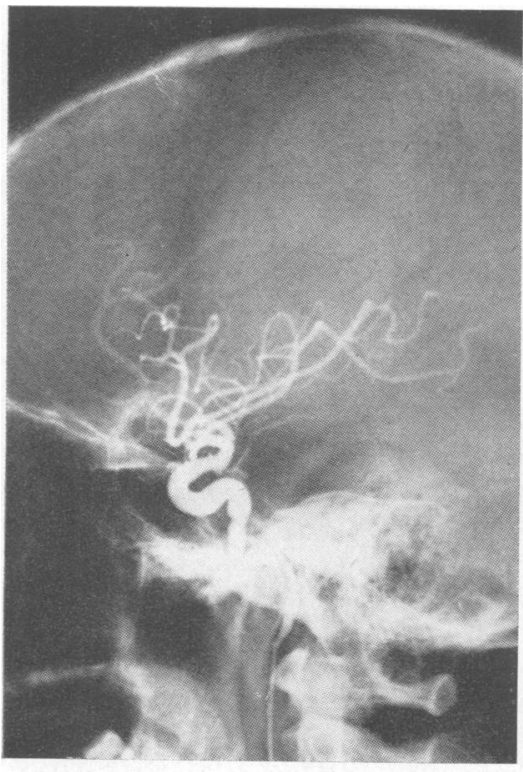

(a)

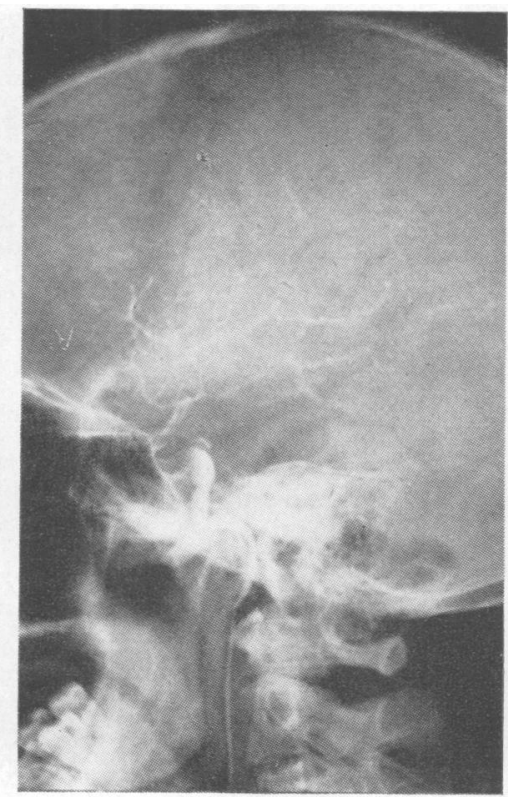

(b)

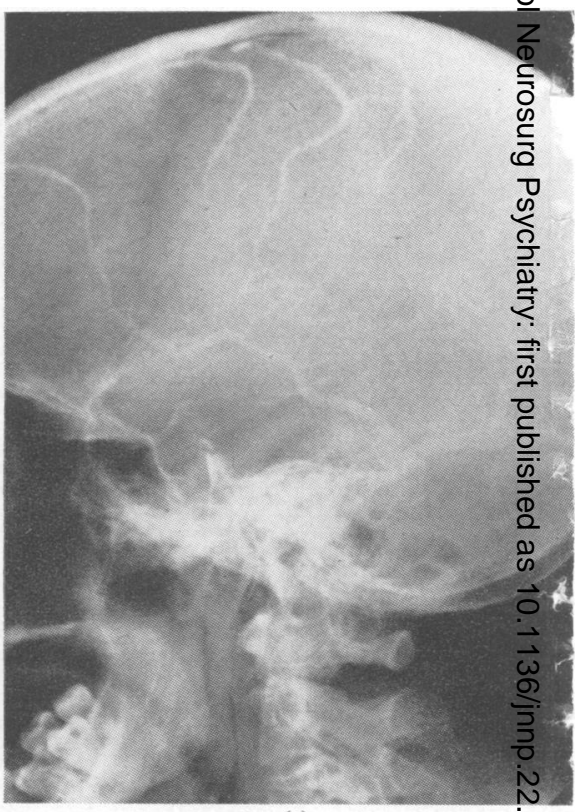

$\nabla \dot{\varphi}$

D.

FIG. 7.-Internal carotid arteriogram performed on another patient showing appearances produced by accidental intramural injection.

(a) Note the tip of the catheter rendered opaque by contrast medium. There is filling of the internal carotid artery and its branches. (b) Film taken two seconds after (a). The contrast medium has left the major branches of the internal carotid artery, but some still persists in the internal carotid artery in the neck and its siphon. (c) Film taken four seconds after (a). The $\stackrel{\overbrace{\mathrm{D}}}{\mathrm{a}} \mathrm{O}$ portion of the contrast medium has already rached the cerebral veins. A narrow streak still persist gn the line of the wall of the internal carotid artery in the reck and in its siphon. macroscopic description but was not examined microscopically as this part of the artery had been in decalcifying fluid.

\section{Discussion}

Radiology.-The radiological appearances seen in this case indicate the presence of a dissecting aneurysm of the internal carotid artery. There is some similarity to those seen in thrombosis of the artery and to those seen where atheromatous plaques are present, but in the former condition no contrast medium passes the site of thrombosis and in the latter small, localized filling defects are present in the shadow of contrast medium filling the arterial lumen.

In carotid angiography the contrast medium may occasionally be injected into an artery when the needle point has accidentally penetrated the arterial wall. This, however, gives a characteristic picture. Fig. 7 illustrates the appearances in such a case. The serial films show that the main part of the contrast medium has passed into the cerebral circulation, while a small part has passed along the wall of the vessel, stripping the tissues in its path and producing a double shadow, the translucent area between representing the arterial wall. In such cases the shadow of contrast medium disappears in less than 10 minutes.

Cases of intramural injection of contrast medium have previously been described by Sirois, Lapoint, and Côté (1954) and by de Grood (1954).

Pathology.-Gore $(1952,1953)$ noted two main types of medial degeneration in cases of dissecting aneurysm in the aorta. These were (1) the elastic tissue type, more often seen in people under the age of 40 , in which the elastica was severely damaged, with relative sparing of the muscle fibres, and in which there was an accumulation of basophilic ground substance; and (2) the muscle tissue type where the muscle was severely damaged, with only slight changes in the elastica. Vascularization of the media was a prominent feature in both groups. In our case there was severe damage to the elastica and there was an accumulation of metachromatic material (basophilic ground substance) in the media. In these respects it conforms with the elastic tissue type of medial degeneration.

Vascularization of the media was not present. 
This, and the recent tears in the intima, are evidence that, in this case, the blood in the media came direct from the lumen of the artery and could not have come from any abnormal vascularization of the media. Such a source of haemorrhage has been suggested in other cases of intramural haemorrhage.

The constant finding of medial degeneration in cases of dissecting aneurysm is considered by some authors to be an important factor in association with the development of the condition. It is often difficult to decide whether this medial degeneration antedates or follows the development of the aneurysm. In our case the presence of similar changes in the otherwise healthy carotid artery is evidence that the medial degeneration preceded the aneurysm.

The presence of an increased amount of metachromatic material in the media is thought to be an important early feature of the elastic tissue type of degeneration in man, and it is found constantly in experimental lathyrism in animals (Menzies and Mills, 1957), where dissecting aneurysms are a prominent feature. In this condition, due to an upset in enzyme metabolism, an accumulation of metachromatic material is found widespread throughout bones and the aorta (Menzies and Mills, 1957) and possibly in other tissues. Meyer and Rapport (1950, quoted by Menzies and Mills, 1957) consider this metachromatic material to be chon- droitin sulphate. This substance is also found to be increased in association with amyloid deposits. Our patient had chronic caseating tuberculosis, which is often associated with secondary amyloidosis, and although amyloid deposits were not seen in the kidneys, liver, or spleen, it is always possible that the same process which can give rise to secondary amyloidosis may here have produced an excess of histologically similar abnormal material in the carotid arteries.

\section{Summary}

A case of spontaneous dissecting aneurysm of the internal carotid artery, in a male of 41 years, is presented.

The radiological and pathological findings are discussed.

We are indebted to Dr. John Marshall for permission to present this case, to Professor W. Blackwood and Dr. Hugh Davies for their advice and help, and to Mr. J. Mills and to the staff of the photographic department of the Institute of Neurology for the photographs.

\section{REFERENCES}

Dratz, H. M. and Woodhall, B. (1947).J. Neuropath., 6, 286 Gore, I. (1952). A.M.A. Arch. Path., 53, 142. - (1953). Ibid., 55, 1 .

Grood, M. P. A. M. de (1954). Rev. neurol., 90, 661.

Menzies, D. W., and Mills, K. W. (1957). J. Path. Bact., 73, 223. Shennan, T. (1934). Med. Res. Coun. Spec. Rep. Series, No. 193. Sirois, J., Lapointe, H., and Côté, P. E. (1954). J. Neurosurg., 11, 